\title{
ANÁLISE COMPARATIVA ENTRE VÁRIOS MÉTODOS DE QUANTIFICAÇÃO DE HEMICELULOSES DA MADEIRA DE EUCALIPTO
}

\section{Lívia P. S. Palmeiras}

Escola Superior de Agricultura "Luiz de Queiroz", Universidade de São Paulo, CP 9, 13418-900 Piracicaba - SP, Brasil

Andréia S. Magaton

Centro de Ciências Exatas e Tecnológicas, Universidade Federal do Recôncavo da Bahia, 44380-000 Cruz das Almas - BA, Brasil Jorge L. Colodette e Ana Márcia Macedo Ladeira Carvalho*

Departamento de Engenharia Florestal, Universidade Federal de Viçosa, 36570-000 Viçosa - MG, Brasil

Recebido em 20/5/09; aceito em 25/2/10; publicado na web em 6/5/10

\begin{abstract}
COMPARATIVE ANALYSIS BETWEEN SEVERAL METHODS OF QUANTIFICATION OF EUCALYPTUS WOOD HEMICELLULOSES. Pulp hemicelluloses can be extracted with $\mathrm{NaOH}$ and quantified by colorimetric and gravimetric techniques. However the most usual methods to measure eucalyptus pulp hemicelluloses have been through the pentosan method or through xylan analyses by GC or HPLC techniques. In this study a comparison was made between the more traditional methods and indirect method of $\mathrm{NaOH} 5 \%$ extraction followed by colorimetric analyses. It was observed that the content of $\mathrm{NaOH} 5 \%$ extract correlates very well with pulp xylan content and reasonably well with the pentosan content. It is concluded that the $5 \% \mathrm{NaOH}$ solubility method can be used in replacement of the other two, since it is faster, simpler and less costly to carry out than the others.
\end{abstract}

Keywords: xylan; pentosan; solubility.

\section{INTRODUÇÃO}

Como as propriedades dos materiais lignocelulósicos e dos produtos com eles formados dependem da presença e do teor relativo dos seus diversos constituintes, a identificação e avaliação quantitativa destes é de grande importância. Por isso, há uma preocupação contínua, por parte das empresas, em desenvolver e aperfeiçoar os procedimentos analíticos para a sua determinação.

As substâncias da madeira que têm despertado grande interesse mundial são as hemiceluloses, um dos principais constituintes da madeira, que estão presentes entre as fibrilas de celulose na parede celular. ${ }^{1,2}$ Em conexão com a produção de polpa celulósica, as hemiceluloses desempenham papel importante, pois sua preservação além de aumentar o rendimento em produção de polpa, facilita a fabricação do papel, possibilitando maior hidratação da fibra e, com isso, economiza-se energia durante o refino da polpa. ${ }^{3,4}$

As hemiceluloses podem ser extraídas da polpa celulósica e quantificadas indiretamente pela medição de solubilidade da polpa de celulose em soluções de hidróxido de sódio em determinadas concentrações. ${ }^{5}$ Em se tratando das espécies de folhosas, o material removido por solução de hidróxido de sódio $5 \%$ consiste, praticamente, de xilanas (Figura 1).

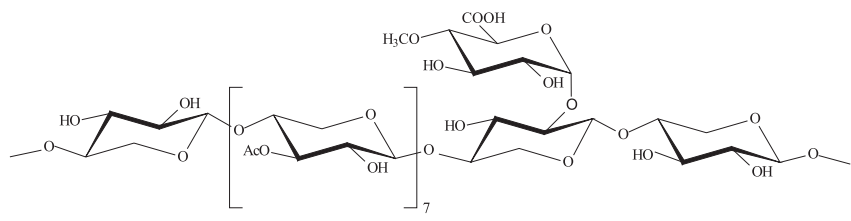

Figura 1. Estrutura química parcial das xilanas

Os métodos utilizados para a medição de hemiceluloses em polpas de fibra curta têm sido o de pentosanas e o de xilanas por cromatografia líquida e/ou gasosa. Porém, essas técnicas demandam tempo e

*e-mail: ana.marcia@ufv.br custo. Por isso, nesse estudo foi feita uma análise comparativa entre os métodos tradicionais (pentosanas e análise direta de xilanas por HPLC) e o método indireto de extração por $\mathrm{NaOH} 5 \%$, visto que este é mais vantajoso em custo e tempo. Deve ser ressaltado que o valor de pentosanas não corresponde ao teor de hemiceluloses no material lignocelulósico. ${ }^{5}$ Mas no caso de madeiras de eucalipto, o teor de pentosanas se aproxima muito do teor de hemiceluloses, uma vez que na madeira de eucalipto estas são compostas principalmente de pentoses. ${ }^{6-9}$ Assim, para polpa Kraft branqueada de eucalipto, o método de pentosanas produz resultados praticamente iguais ao método de análise direta de xilanas, evidenciando a ausência de arabinose e de outras pentoses nestas polpas. Isto ocorre porque, entre as hemiceluloses, somente as xilanas resistem à polpação Kraft e ao branqueamento ECF. ${ }^{10,11}$

O objetivo desse estudo foi comparar os métodos tradicionais (pentosanas e análise direta de xilanas por HPLC) e o método indireto de extração por $\mathrm{NaOH} 5 \%$ seguido de análise colorimétrica do extrato, para sete amostras de polpa de eucalipto branqueadas. Para isso, as amostras foram caracterizadas quanto à percentagem de xilanas, lignina, pentosanas e ácidos hexenurônicos. As polpas também foram caracterizadas quanto ao número kappa e viscosidade.

\section{PARTE EXPERIMENTAL}

Foram utilizadas para essa análise sete amostras de polpas Kraft industriais branqueadas de eucalipto. As características iniciais das amostras estão apresentadas na Tabela 1 .

Foram confeccionadas folhas laboratoriais, das respectivas amostras, usando uma formadora do tipo Tappi. As folhas foram formadas e acondicionadas em ambiente climatizado com temperatura de $23 \pm$ $1{ }^{\circ} \mathrm{C}$ e umidade relativa do ar de $50 \pm 2 \%$, posteriormente moídas em moinho do tipo IKAR A 11 basic. Em seguida, realizou-se a pesagem das amostras para determinação do teor de umidade.

Foram pesados, aproximadamente, 1,5 $\mathrm{g}$ de polpa, com precisão de $0,02 \mathrm{~g}$, que foram transferidos, para um erlenmeyer de $250 \mathrm{~mL}$. Adicionaram-se por meio de uma pipeta, $100 \mathrm{~mL}$ de solução de 
Tabela 1. Características iniciais das polpas

\begin{tabular}{|c|c|c|c|c|}
\hline \multirow[b]{2}{*}{ Polpas } & \multicolumn{4}{|c|}{ Características iniciais das polpas } \\
\hline & Número Kappa & $\begin{array}{c}\text { Viscosidade } \\
\left(\mathrm{dm}^{3} / \mathrm{kg}\right)\end{array}$ & Lignina $(\%)$ & $\begin{array}{c}\text { HexA's } \\
(\mathrm{mmol} / \mathrm{kg})\end{array}$ \\
\hline 1 & 1,25 & 689 & 0,080 & 7 \\
\hline 2 & 0,75 & 758 & 0,011 & 6,6 \\
\hline 3 & 1,20 & 723 & 0,068 & 7,5 \\
\hline 4 & 0,60 & 757 & 0,041 & 3,1 \\
\hline 5 & 0,62 & 889 & 0,072 & 1,3 \\
\hline 6 & 0,65 & 842 & 0,071 & 2,2 \\
\hline 7 & 2,00 & 875 & 0,116 & 11,7 \\
\hline
\end{tabular}

hidróxido de sódio $5 \%$ (a $25 \pm 0,2^{\circ} \mathrm{C}$ ) e manteve-se o conjunto a 25 $\pm 0,2^{\circ} \mathrm{C}$, em banho termostático, por $60 \mathrm{~min}$, contados a partir da adição da solução de hidróxido de sódio, misturando frequentemente com bastão de vidro. Após exatamente $60 \mathrm{~min}$, filtrou-se a mistura em sucção amena em cadinho de vidro sinterizado (porosidade média), recebendo o filtrado em kitazato de $500 \mathrm{~mL}$, limpo e seco, desprezando os primeiros 10 a $20 \mathrm{~mL}$ e não deixando passar ar através do resíduo. Em seguida, pipetaram-se $20 \mathrm{~mL}$ do filtrado e $10 \mathrm{~mL}$ da solução de dicromato de potássio $0,5 \mathrm{~N}$ e transferiu-se para um erlenmeyer. Em capela de exaustão adicionaram-se, cuidadosamente de forma lenta e gradual e sob leve agitação, $45 \mathrm{~mL}$ de ácido sulfúrico p.a. com aparelhagem volumétrica. Esfriou-se a solução por 15 min e adicionaram-se $50 \mathrm{~mL}$ de água deixando o sistema chegar à temperatura ambiente. Homogeneizou-se levemente a solução e esta foi levada ao banho termostático por 5 min a $25 \pm 0,2^{\circ} \mathrm{C}$. Retirou-se a solução do banho, adicionaram-se gotas de indicador ferroína e titulou-se com solução de sulfato ferroso $0,1 \mathrm{~N}$ até o aparecimento da coloração púrpura estável por alguns segundos. O cálculo da solubilidade em hidróxido de sódio foi obtido da seguinte forma:

$\mathrm{S}_{5 \%}(\%)=\left(\mathrm{V}_{2}-\mathrm{V}_{1}\right) * \mathrm{~N} * 6,85 * \mathrm{~F} * 100 * 100 /(\mathrm{A} * \mathrm{~W} * 1000)$

onde: $\mathrm{S}_{5 \%}(\%)$ é o percentual de material solúvel em $\mathrm{NaOH} 5 \%$; $\mathrm{V}_{2}$ é o volume de sulfato ferroso amoniacal, em $\mathrm{mL}$, consumido na titulação da prova em branco; $V_{1}$ é o volume de sulfato ferroso amoniacal, em mL, consumido na titulação; $\mathrm{N}$ é a normalidade da solução de sulfato ferroso amoniacal; teoricamente, 1 miliequivalente de $\mathrm{K}_{2} \mathrm{Cr}_{2} \mathrm{O}_{7}$ corresponde a $6,75 \mathrm{mg}$ de celulose e outras hexoses, e 6,60 mg de pentoses. Nas condições do teste, menos oxidante é consumido e 1 miliequivalente corresponde a $6,85 \mathrm{mg}$ de celulose e outros carboidratos dissolvidos; F é o fator da solução de sulfato ferroso amoniacal; 100 é o fator de conversão para porcentagem; 100 é o $100 \mathrm{~mL}$ da solução de $\mathrm{NaOH} 5 \%$; A, é o volume do filtrado da polpa, em mL, utilizado na oxidação; $\mathrm{W}$, é o valor da massa usada no teste; 1000 é o fator de conversão de g para mg.

Os demais métodos de análise utilizados foram os padrões TAPPI, de acordo com a Tabela 2. Todas as análises foram realizadas em duplicata.

Tabela 2. Normas utilizadas

\begin{tabular}{lc}
\hline Parâmetro analisado & Metodologia \\
\hline Confecção de folhas $^{12}$ & TAPPI - 205 \\
Número kappa $^{12}$ & TAPPI - 236 \\
Viscosidade $^{12}$ & TAPPI - 230 om 82 \\
Pentosanas $^{12}$ & TAPPI T 223 ts-63 \\
Lignina Klason $^{13}$ & Gomide e Demuner,1986 \\
Hexenurônico $^{14}$ & Vuorinen et al., 1996 \\
Xilanas $^{15}$ & Kaar et al., 1991 \\
\hline
\end{tabular}

\section{RESULTADOS E DISCUSSÃO}

As percentagens de pentosanas, xilanas e solubilidade em $\mathrm{NaOH}$ $5 \%$ podem ser observadas na Tabela 3 . Já a relação entre as percentagens de pentosanas e xilanas está representada na Figura 2.

Tabela 3. Percentagens de pentosanas e xilanas

\begin{tabular}{cccc}
\hline Polpa & $\mathrm{S}_{5}(\%)$ & Xilanas $(\%)$ & Pentosanas $(\%)$ \\
\hline 1 & 9,3 & 14,80 & 14,00 \\
2 & 9,1 & 14,30 & 14,50 \\
3 & 8,9 & 14,40 & 14,50 \\
4 & 8,6 & 13,90 & 14,00 \\
5 & 10,6 & 15,90 & 15,40 \\
6 & 8,2 & 13,70 & 13,60 \\
7 & 8,4 & 13,80 & 13,90 \\
\hline
\end{tabular}

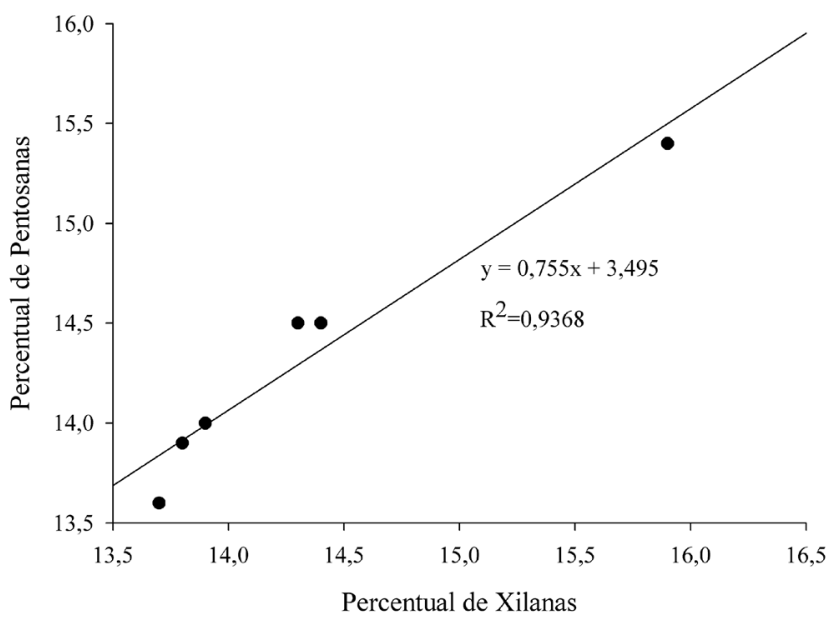

Figura 2. Correlação entre as percentagens de pentosanas e xilanas

As percentagens de pentosanas e xilanas apresentaram boa correlação entre si $\left(\mathrm{R}^{2}=0,94\right)$. Um teor de $15,3 \%$ de xilanas equivale a $15,1 \%$ de pentosanas. Portanto, estas duas técnicas podem ser usadas intercambiavelmente para medir o teor de hemiceluloses de polpa branqueada de eucalipto, já que a presença de outras hemiceluloses nestas polpas é negligível. ${ }^{16}$

A correlação $\left(R^{2}=0,98\right)$ entre a solubilidade da polpa de celulose em hidróxido de sódio 5\% e o teor de xilanas foi significativa (Figura 3). Isso é justificado pelo fato de que as xilanas são as hemiceluloses resistentes ao processo de polpação Kraft e ao branqueamento ECF em polpas de eucalipto.

Assim, pela Figura 3 uma solubilidade de $10 \%$ de hemiceluloses em solução de hidróxido de sódio $5 \%$ equivale a $15,3 \%$ de xilanas, considerando polpas branqueadas de eucalipto que só apresentam este tipo de hemiceluloses.

Da mesma forma, é possível observar, pela Figura 4, que houve uma boa correlação entre solubilidade em soda $5 \%$ e pentosanas $\left(\mathrm{R}^{2}=0,83\right)$. Uma solubilidade de $10 \%$ em hidróxido de sódio $5 \%$ equivale a $15,1 \%$ de pentosanas. Isso confirma a predominância das pentoses como hemiceluloses das madeiras de eucalipto.

Em princípio, polpas contendo alto teor de xilanas deveriam conter também um alto teor de ácidos hexenurônicos, já que esses ácidos estão associados às xilanas. Porém, o conteúdo de ácidos hexenurônicos (HexA's) da polpa depende grandemente da forma como ela foi produzida, isto é, dos métodos de polpação e branqueamento empregados. ${ }^{17,18}$ A Figura 5 mostra uma correlação muito baixa entre 


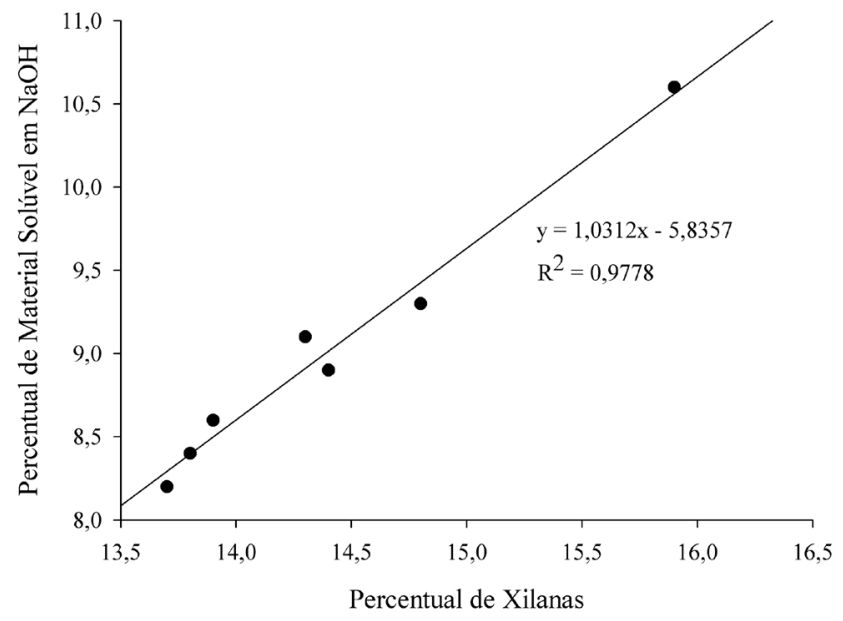

Figura 3. Correlação entre material solúvel em $\mathrm{NaOH} 5 \%$ e xilanas

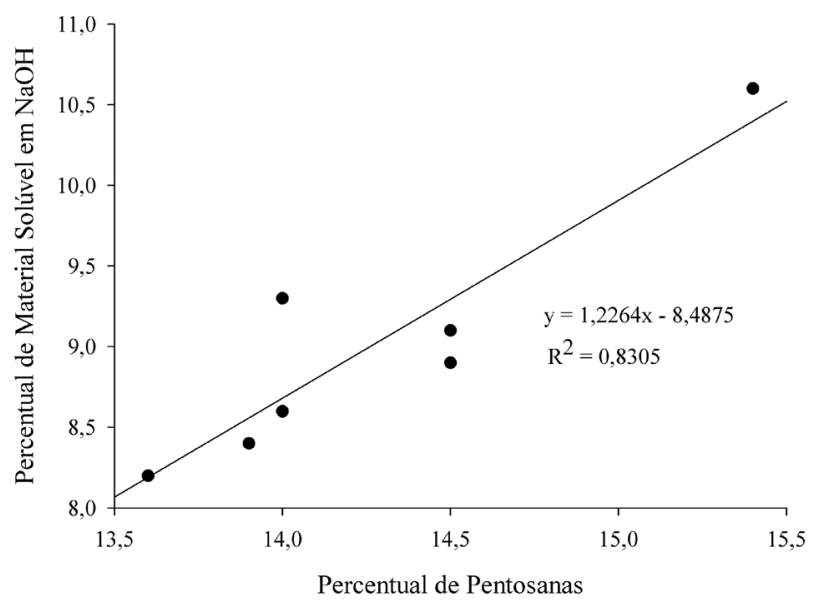

Figura 4. Correlação entre material solúvel em $\mathrm{NaOH} 5 \%$ e pentosanas

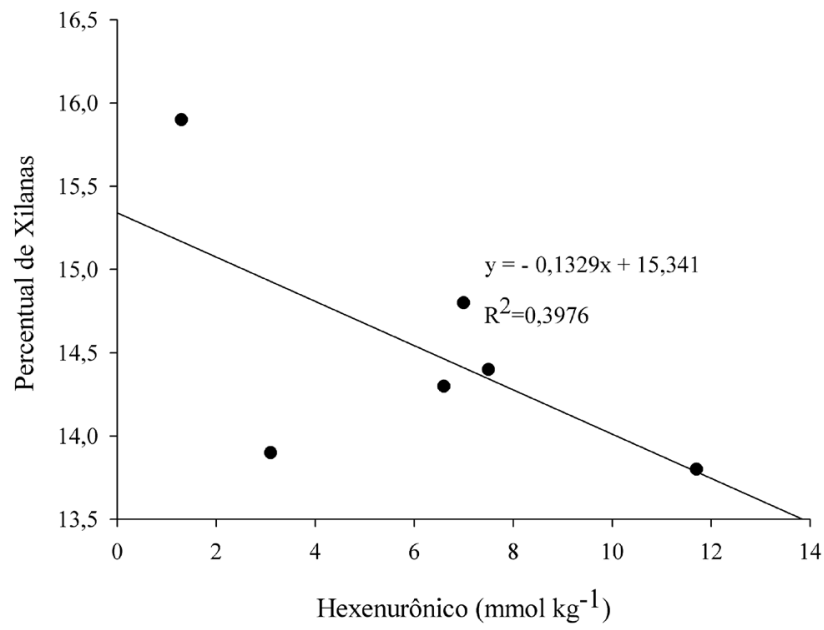

Figura 5. Correlação entre percentual de xilanas e ácidos hexenurônicos os teores de xilanas e de ácidos hexenurônicos, realçando as diferenças marcantes nas tecnologias de produção das várias amostras de polpa empregadas neste estudo.

\section{CONCLUSÃO}

O método de solubilidade da polpa celulósica em solução de hidróxido de sódio $5 \%$ mostrou-se adequado para a quantificação de hemiceluloses de polpa branqueada de eucalipto. Por ser um método simples, rápido e de baixo custo, ele se apresenta como boa alternativa aos tradicionais métodos de pentosanas e de análise direta de xilanas por HPLC.

\section{AGRADECIMENTOS}

Ao Conselho Nacional de Desenvolvimento Científico e Tecnológico $(\mathrm{CNPq})$ pelo apoio financeiro.

\section{REFERENCIAS}

1. Evtuguin, D. V.; Tomás, J. L.; Silva, A. M. S.; Pascoal Neto, C.; Carbohydr. Res. 2003, 338, 597.

2. Ferreira, V. F.; Rocha, D. R.; Silva, F. C.; Quim. Nova 2009, 32, 623.

3. Evtuguin, D. V.; Pascoal Neto, C.; Resumos do III Colóquio Internacional sobre Celulose Kraft de Eucalipto, Belo Horizonte, Brasil, 2007.

4. Magaton, A. S.; Colodette, J. L.; Gouvêa, A. F. G.; Gomide, J. L.; Muguet, M. C. S.; Pedrazzi, C.; Tappi J. 2009, 9, 32.

5. Browning, B. L.; Methods of Wood Chemistry, $1^{\text {st }}$ ed., Interscience Publishers: New York, 1967.

6. Lee, Y. Y.; Xiang, Q.; Torget, R. W.; Appl. Biochem. Biotechnol. 2004, 113-116, 1127.

7. Neto, C.; Evtuguin, D.; Pinto, P.; O Papel 2005a, 66, 17.

8. Neto, C.; Evtuguin, D.; Pinto, P.; Silvestre, A.; Freire, C.; Proceedings Appita Annual Conference 2005b, 2: 431.

9. Ona, T.; Sonoda, T.; Ito, K.; Shibata, M.; Holtzforschung 1997, 51, 396.

10. Pinto, P. C.; Evtuguin, D. V.; Pascoal Neto, C.; Carbohydr. Polym. 2005, $60,489$.

11. Pedrazzi, C.; Tese de Doutorado, Universidade Federal de Viçosa, Brasil, 2009.

12. TAPPI; TAPPI Test Methods, Tappi Press: Atlanta, 1998.

13. Gomide, J. L.; Demuner, B. J.; O Papel 1986, 47, 36.

14. Vuorinen, T.; Teleman, A.; Fagerstrom, P.; Buchert, J.; Tenkanen, M.; Proceedings Pulp Bleaching Conference, Washington D.C., USA, 1996.

15. Karr, W. E.; Cool, L. G.; Merriman, M. M.; Brink, D. L.; J. Wood Chemistry. Technology 1991, 11, 447.

16. Ferreira, C. R.; Fantini Jr., M.; Colodette, J. L.; Oliveira, R. C.; Gomide, J. L.; Carvalho, A. M. M. L.; Science Florestalis 2006, 71, 9.

17. Buchert, J.; Laine, J.; Tenkanen, M.; Vuorinen, T.; Viikari, L.; Proceedings $9^{\text {th }}$ International Symposium Wood Pulping Chemistry Proceedings. Technical Section, CPPA, Montreal, Canada, 1997.

18. Ventorim, G.; Caraschi, J. C.; Colodette, J. L.; Gomide, J. L.; Quim. Nova 2009, 32, 373 . 